\title{
Repercussões do tabagismo na ultra-sonografia da placenta e a doplervelocimetria útero-placentária
}

\author{
Effects of maternal smoking on placental ultrasound and uterine-placental Doppler \\ Sebastião José Saraiva Filho' ${ }^{1}$, Antonio Fernandes Moron², Luiz Antonio Bailão ${ }^{3}$, \\ Maria Christina dos Santos Rizzi ${ }^{4}$, Mary Uchyiama Nakamura ${ }^{5}$
}

\section{RESUMO}

Objetivo: avaliar as repercussões ultra-sonográficas do tabagismo materno na placenta, com ênfase no seu grau de maturação (calcificação), e correlacionar estes achados com o padrão hemodinâmico útero-placentário com uso da doplervelocimetria das artérias uterinas e umbilicais. Métodos: estudo prospectivo do tipo coorte envolvendo 244 gestantes, sendo 210 não-fumantes e 34 fumantes. Cada paciente submeteu-se a quatro exames ecográficos sendo o primeiro até a $16^{\mathrm{a}}$ semana, para datar a gestação. Subseqüentemente, na $28^{\mathrm{a}}, 32^{\mathrm{a}}$ e $36^{\mathrm{a}}$ semana, foram efetivadas novas ultra-sonografias para biometria fetal, avaliação da ecotextura placentária e estudo doplerfluxométrico das artérias uterinas e umbilicais. $\mathrm{O}$ achado ultra-sonográfico de placenta grau III antes da $36^{\mathrm{a}}$ semana foi considerado como calcificação precoce. Para análise estatística foram aplicados os testes do $\chi^{2}$ e o exato de Fisher na avaliação comparativa dos graus placentários, e o teste de Mann-Whitney para o índice de resistência das artérias uterinas e umbilicais. Resultados: não foram observadas diferenças significantes na freqüência de placenta grau III e no índice de resistência das artérias uterinas entre as fumantes e não fumantes, nas diferentes idades gestacionais. O índice de resistência da artéria umbilical na $32^{\mathrm{a}}$ semana foi significantemente maior nas tabagistas $(0,64$ versus $0,61, \mathrm{p}<0,05)$. Conclusões: não se evidenciou associação do tabagismo com aceleração da maturação placentária. Neste estudo o vício de fumar esteve associado a alterações vasculares da circulação útero-placentária apenas na $32^{\mathrm{a}}$ semana de gravidez.

PALAVRAS-CHAVE: Tabaco; Complicações da gravidez; Placenta/ultrasonografia; Circulação placentária; Fluxometria por laser-doppler; Tabagismo

\section{ABSTRACT}

Purpose: the study the effects of maternal cigarette smoking during pregnancy on placental maturation (calcifications) and the placental-uterine circulation, evaluated through umbilical and uterine Doppler. Methods: prospective cohort study involving 244 pregnant women, 210 of them non-smokers and 34 smokers. Participants were submitted to four serial sonograms. The first was performed up to the 16th week of pregnancy to determine gestational age, and the other three at 28, 32 and 36 weeks for fetal biometry, evaluation of placental texture and Doppler studies of the uterine and umbilical arteries. Premature placental calcification was defined as grade III before 36 weeks. The $\chi^{2}$ and Fisher exact tests were used to compare placental grading, and the Mann-Whitney test to evaluate the resistance index of uterine and umbilical arteries. Results: the frequency of grade III placenta and the resistance of the uterine arteries did not differ significantly between smokers and non-smokers, at all gestational ages. Umbilical artery Doppler was significantly higher in smokers than in non-smokers at 32 weeks. Conclusions: no association was found between cigarette smoking and premature placental calcification. Smoking was associated with increased umbilical artery resistance at 32 weeks.

KEYWORDS: Tobacco; Pregnancy complications; Placenta/ultrasonography; Placental circulation; Laser-doppler flowmetry; Smoking

Diagnosis - Centro de Treinamento em Ultra-sonografia, Ribeirão Preto - São Paulo (SP), Brasil.

1 Médico Assistente do Diagnosis - Centro de Treinamento em Ultra-sonografia, Ribeirão Preto - São Paulo (SP), Brasil.

2 Professor Titular do Departamento de Obstetrícia da Universidade Federal de São Paulo - UNIFESP - São Paulo (SP), Brasil.

3 Diretor do Diagnosis - Centro de Treinamento em Ultra-sonografia, Ribeirão Preto - São Paulo (SP), Brasil.

4 Vice-Diretora do Diagnosis - Centro de Treinamento em Ultra-sonografia, Ribeirão Preto - São Paulo (SP), Brasil.

5 Chefe do Departamento de Obstetrícia da Universidade Federal de São Paulo - UNIFESP - São Paulo (SP), Brasil

Correspondência: Sebastião José Saraiva Filho

Av. Caramuru, 732, Apto. 34 - Residencial Ilha de Bali - Jardim República - 14030-000 - Ribeirão Preto - SP - Telefone/Fax: (16) 3911.4435 Cel.: (16) 9962.7813 - e-mail: sebastiaosaraiva@uol.com.br

Recebido em: 23/9/2006 


\section{Introdução}

Estima-se que mais de 30\% das mulheres em idade reprodutiva sejam tabagistas e que apenas uma em cada cinco abandone o vício ao engravidar ${ }^{1}$. Dados recentes indicam que um terço das gestantes americanas e um quarto das brasileiras são tabagistas ${ }^{2}$. O hábito de fumar acarreta riscos fetais decorrentes do amadurecimento placentário precoce ${ }^{1}$ e redução do aporte nutricional, podendo provocar restrição do crescimento fetal ${ }^{3}$. Adicionalmente, a aceleração da maturidade placentária tem sido associada a aumento na incidência de descolamento prematuro da placenta ${ }^{1}$. Além de acarretar riscos perinatais, o tabagismo materno pode também afetar o desenvolvimento mental, intelectual e comportamental das crianças expostas na vida fetal ao fumo ${ }^{4}$.

$\mathrm{Na}$ literatura existem diversos trabalhos individuais sobre o efeito do tabagismo no amadurecimento placentário ${ }^{5}$ e na circulação útero-placentária $^{6,7}$, porém nenhum estudo sobre esses dois aspectos analisados em conjunto, nas mesmas pacientes. Este trabalho visou estudar em gestantes tabagistas o processo de maturação placentária e, ao mesmo tempo, correlacionar estes achados com os resultados da dopplervelocimetria da circulação útero-placentária.

\section{Métodos}

Este foi um estudo coorte prospectivo desenvolvido entre abril de 2004 e junho de 2005, com 244 gestantes residentes na região de Ribeirão Preto, São Paulo. O trabalho foi aprovado pela Comissão de Ética em Pesquisa da Universidade Federal de São Paulo e todas as pacientes assinaram termo de consentimento informado. As pacientes eram atendidas pelo Sistema Único de Saúde (SUS) e foram referidas para realizar exames ultra-sonográficos obstétricos no Diagnosis - Centro de Treinamento em Ultra-sonografia. Foram consideradas elegiveis para o estudo todas as gestantes com idade gestacional inferior a 17 semanas, tabagistas ou não, com feto único e vivo, sem intercorrências clínicas e/ou obstétricas que pudessem alterar o desenvolvimento placentário e/ou a hemodinâmica útero-placentária. Foram excluídas as pacientes com ameaça de aborto, hipertensão arterial, diabetes melito, descolamento prematuro de placenta, gravidez ectópica e/ou heterotópica, malformações fetais ou neoplasia trofoblástica gestacional. Foram também excluídas as gestantes que, por qualquer motivo, perderam um dos quatro exames previstos. Das 828 pacientes inicialmente cadastradas e submetidas ao primeiro exame ecográfico, 584 foram excluídas por não cumprirem os critérios de inclusão, encerrando-se o estudo com 244 gestantes.

As gestantes foram divididas em dois grupos: não fumantes $(n=210)$ e fumantes $(n=34)$. As tabagistas foram classificadas em três categorias, conforme o número de cigarros consumidos por dia: leve (1 a 5 cigarros/dia, $n=9)$ ), moderada (6 a 10 cigarros/dia, $n=18$ ), acentuada (mais de 10 cigarros/dia, n=7). Fumantes passivas não foram consideradas neste estudo.

Cada paciente foi submetida a quatro exames ultra-sonográficos seriados. O primeiro foi realizado até a $16^{\mathrm{a}}$ semana, visando confirmar a idade gestacional. O tempo de amenorréia referido pela paciente foi confirmado pela ultra-sonografia, pela biometria fetal (comprimento cabeça-nádegas, diâmetro biparietal, circunferência craniana, circunferência abdominal e comprimento do fêmur e do úmero). $\mathrm{Na} 28^{\mathrm{a}}, 32^{\mathrm{a}}$ e $36^{\mathrm{a}}$ semana, cada paciente submeteuse a novas ultra-sonografias para biometria fetal, análise da ecotextura placentária e estudo doplervelocimétrico das artérias uterinas e umbilicais, pela mensuração do índice de resistência (IR).

A cada exame a placenta foi classificada em graus (0 a III), segundo os critérios estabelecidos por Grannum et al. ${ }^{8}$. A placenta era classificada como grau 0 quando apresentava placa corial lisa e ecotextura homogênea; grau I quando a placa corial era ondulada e a ecotextura apresentava pontos ecogênicos (calcificações) esparsos; grau II quando apresentava calcificações em forma de "virgula", da placa corial em direção à basal, e grau III quando as calcificações esboçavam imagens em anel.

O IR das artérias uterinas foi baseado na média dos valores encontrados em entre ambas (direita e esquerda). O IR das artérias umbilicais contemplou a média dos valores encontrados nas porções insonadas (placentária, alça livre e abdominal). O estudo doplervelocimétrico das artérias uterinas e umbilicais foi realizado uma única vez a cada exame.

Todos as ultra-sonografias foram feitas por um único examinador (o primeiro autor), utilizando o aparelho GE Ultrasound, modelo Logic 400 Pro, com transdutores de $7,5 \mathrm{MHz}$ (para os exames transvaginais) e de 3,5 MHz (para os exames transabdominais).

O tamanho amostral foi calculado supondose população de gestantes infinita e por estudos anteriores com a estimativa da proporção dos casos (gestantes fumantes) em $25 \%$ da população. O erro máximo de estimativa da proporção foi fixado em $6 \%$ (precisão absoluta) com 95\% de confiança $(\alpha=5,0 \%)$. Encontramos o tamanho amostral de 204 gestantes. 
As informações obtidas foram codificadas e digitadas em um banco de dados, utilizando-se o programa Epi-Info 6.0. Posteriormente, essas informações foram submetidas à análise estatística, utilizando-se o programa STATA 6.0. O nível de significância foi fixado em $5 \%(a \leq 0,05)$ para a análise estatística dos dados.

Avaliou-se, comparativamente, a diferença da maturação placentária entre os grupos de gestantes não fumantes e fumantes nas diferentes idades gestacionais, fazendo-se o mesmo em relação aos valores médios do IR das artérias uterinas e umbilicais. Para avaliar a associação do grau de maturação placentária com o vício de fumar, foram utilizados os testes do $\chi^{2}$ e de Fischer. O teste de Mann-Whitney foi empregado para comparação do IR entre os dois grupos nas diferentes idades gestacionais.

A média de idade das 244 gestantes foi de 23,8 anos ( $\pm 5,6$ anos), variando de 13 a 38 anos. A idade média das fumantes e não fumantes foi semelhante $(26,0$ versus $23,5, p=0,054)$. A maioria $(56,6 \%)$ das pacientes era nulipara $(n=138) ; 19,2 \%$ eram primiparas e $24,2 \%$ eram multiparas.

\section{Resultados}

Apesar de se evidenciar tendência para graus placentários mais avançados entre as fumantes, não foram encontradas diferenças significantes na distribuição dos diversos graus placentários entre fumantes e não fumantes na $28^{\mathrm{a}}, 32^{\mathrm{a}}$ e $36^{\mathrm{a}}$ semana de gestação (Tabela 1).
A soma das freqüências de placentas grau II e III, na $28^{\mathrm{a}}, 32^{\mathrm{a}}$ e na $36^{\mathrm{a}}$ semana, foi de 4,8 , 31,4 e $68,6 \%$ para as não fumantes e 5,9, 38,2 e $76,5 \%$ entre as fumantes, respectivamente. Estas diferenças não foram significantes.

Apesar de pequenas diferenças na $28^{\mathrm{a}}$ e $32^{\mathrm{a}}$ semana, a média do IR das artérias uterinas das gestantes fumantes comparada à das não fumantes não mostrou diferenças significantes. O IR médio das artérias umbilicais das gestantes fumantes mostrou-se maior em todas as idades gestacionais (0,66, 0,64 e 0,59), em comparação com as não fumantes $(0,65,0,61$ e 0,57$)$, mas somente na $32^{a}$ semana a diferença foi estatisticamente significante $(0,64$ versus 0,61$)$ (Tabela 2).

\section{Discussão}

A literatura tem documentado o efeito do fumo sobre o amadurecimento precoce da placenta. Comparadas às não fumantes, as gestantes tabagistas têm maior prevalência de placentas grau III e o seu surgimento é mais precoce. O achado de placenta grau III antes da $37^{\mathrm{a}}$ semana é duas vezes mais freqüente entre as fumantes do que nas não fumantes (36 versus 14\%) ${ }^{9}$. A placenta grau III é identificada, em média, em gestações com 34,4 semanas nas fumantes e com 38,3 semanas entre as não fumantes ${ }^{1}$. Confirmando esses achados, um trabalho naciona ${ }^{10}$ relatou que nas fumantes o grau III era identificado em média com 31,9 semanas, versus 35,8 semanas nas não fumantes, e que $4,3 \%$ das fumantes já apresentavam placentas

Tabela 1 - Frequência de graus placentários em diferentes idades gestacionais conforme o uso ou não de cigarro.

\begin{tabular}{|c|c|c|c|c|c|c|}
\hline \multirow{2}{*}{ Graus } & \multicolumn{3}{|c|}{ Não fumantes (\%) } & \multicolumn{3}{|c|}{ Fumantes (\%) } \\
\hline & $28^{\mathrm{a}} \mathrm{sem}$ & $32^{\mathrm{a}}$ sem & $36^{\mathrm{a}}$ sem & $28^{\mathrm{a}}$ sem & $32^{\mathrm{a}} \mathrm{sem}$ & $36^{\mathrm{a}} \mathrm{sem}$ \\
\hline 0 & 32,9 & 1,4 & - & 38,2 & - & - \\
\hline I & 62,3 & 67,2 & 31,4 & 55,9 & 61,8 & 23,5 \\
\hline$\|$ & 4,8 & 29,0 & 54,3 & 5,9 & 32,3 & 55,9 \\
\hline III & - & 2,4 & 14,3 & - & 5,9 & 20,6 \\
\hline
\end{tabular}

Grau 0: placa corial lisa e ecotextura homogênea.

Grau I: placa corial ondulada e ecotextura com algumas calcificações esparsas;

Grau II: calcificações em "vírgula", da placa corial em direção à basal;

Grau III: calcificações esboçam imagens em anel.

Tabela 2 - Comparação das médias dos índices de resistência (IR) média das artérias uterinas e umbilicais entre gestantes não-fumantes e fumantes em diferentes idades gestacionais.

\begin{tabular}{|c|c|c|c|c|c|c|}
\hline \multirow[b]{3}{*}{ IR médio } & \multirow{2}{*}{\multicolumn{3}{|c|}{$\begin{array}{c}\text { Não fumantes } \\
\text { Semana }\end{array}$}} & \multirow{2}{*}{\multicolumn{3}{|c|}{$\begin{array}{c}\text { Fumantes } \\
\text { Semana }\end{array}$}} \\
\hline & & & & & & \\
\hline & $28^{\mathrm{a}}$ & $32^{\mathrm{a}}$ & $36^{\mathrm{a}}$ & $28^{\mathrm{a}}$ & $32^{\mathrm{a}}$ & $36^{\mathrm{a}}$ \\
\hline Artéria uterina & 0,45 & 0,43 & 0,43 & 0,43 & 0,44 & 0,43 \\
\hline Artéria umbilical & 0,65 & $0,61^{*}$ & 0,57 & 0,66 & $0,64^{*}$ & 0,59 \\
\hline
\end{tabular}

Rev Bras Ginecol Obstet. 2006; 28(6): 340-4. 
grau III entre a $26^{\mathrm{a}}$ e a $32^{\mathrm{a}}$ semana. Em grande estudo recente, envolvendo quase duas mil gestantes de baixo risco, 3,8\% das fumantes já apresentavam placenta grau III antes da $36^{\mathrm{a}}$ semana ${ }^{11}$. Ao contrário da maioria, alguns autores ${ }^{12}$ não acreditam que o tabaco leve à aceleração da maturidade placentária, embora aceitem que o fumo se relacione com a restrição do crescimento fetal. Neste estudo, em concordância com a maioria dos outros trabalhos da literatura, a prevalência de placentas grau III antes da $36^{\mathrm{a}}$ semana foi maior entre as fumantes (20,6 versus 14,3\%), apesar de essa diferença não ter sido significante. Possivelmente, o pequeno tamanho amostral (34 gestantes fumantes) e também o reduzido número de tabagistas que consumiam mais de 10 cigarros/dia (7 gestantes) não tenham sido suficientes para a demonstração formal desta associação.

Apesar do seu pequeno tamanho amostral, este estudo, ao contrário de outros ${ }^{9}$ foi do tipo coorte e todos os exames foram feitos por um único examinador, utilizando o mesmo equipamento ultra-sonográfico, o que valida os seus achados.

Além de acelerar a maturação placentária, o tabagismo provoca alterações da circulação materno-fetal. O cigarro contém mais de 4000 substâncias, das quais as mais nocivas ao organismo humano seriam o monóxido de carbono, o cianeto, os hidrocarbonetos policíclicos aromáticos e a nicotina ${ }^{2}$, que é o principal responsável pelas reduções agudas da circulação útero-placentária ${ }^{13}$. A nicotina provocaria vasoconstricção da circulação uterina por ação simpaticomimética, ocasionando hipoxia transitória e diminuição dos movimentos respiratórios fetais ${ }^{14}$. Como efeito imediato da nicotina, há redução significante no fluxo da artéria uterina $^{15}$, ocasionando queda do IR de 0,55 para $0,49^{16}$, o que poderia prejudicar o crescimento fe$\mathrm{tal}^{17}$. O método radioisótopo permitiu identificar a diminuição crônica do fluxo sanguíneo através dos espaços intervilosos placentários nas fumantes ${ }^{18}$, ao passo que a microscopia eletrônica revelou alterações degenerativas do endotélio vascular no cordão

\section{Referências}

1. Pinette MG, Loftus-Brault K, Nardi DA, Rodis JF. Maternal smoking and accelerated placental maturation. Obstet Gynecol. 1989;73(3 Pt 1):379-82.

2. Carmo AV, Silva LGP, Vidigal N, Pereira Júnior JP, Alzuguir JCL. Fumo e gravidez: repercussões hemodinâmicas materna e fetal. J Bras Ginecol. 1996;106(4):95-100.

3. Davies DP, Abernethy M. Cigarette smoking in pregnancy: Associations with maternal weight gain and fetal growth. Lancet. 1976;1(7956):385-7. umbilical de recém-nascidos de fumantes ${ }^{19}$. Estudos dopplervelocimétricos demonstraram alterações, especialmente na artéria umbilical, indicando aumento de resistência ao fluxo sanguíneo ${ }^{2,7}$.

Neste estudo, que analisou o efeito crônico do tabagismo sobre a circulação útero-placentária, observou-se na $32^{\mathrm{a}}$ semana aumento significante do IR da artéria umbilical entre as fumantes, quando comparadas com as não fumantes, apesar de os valores de ambos os grupos estarem ainda dentro dos parâmetros de normalidade.

Por outro lado, com a doplervelocimetria das artérias uterinas não se demonstrou diferença significante entre os dois grupos. Estudo com desenho semelhante ${ }^{20}$ relatou que ao longo da gestação, as fumantes tinham IR das artérias uterinas e umbilicais mais elevados do que as não fumantes, com diferenças significantes na $36^{\mathrm{a}}$ e $28^{\mathrm{a}}$ semana, para os respectivos vasos. Outros contestam esses achados e relatam que os efeitos do fumo sobre a hemodinâmica útero-placentária são mais agudos do que contínuos ${ }^{5,21}$.

Além da quantidade de cigarros por dia, seria também importante especificar qual a concentração de nicotina e monóxido de carbono nos cigarros consumidos pelas gestantes, dado este não avaliado neste e nem em outros estudos ${ }^{20,21}$.

Confirmando achados de outros autores ${ }^{6,7}$, os resultados deste estudo parecem indicar que o tabagismo afetaria mais a circulação umbilical do que a uterina. Estes achados talvez sugiram que os vasos neoformados sejam mais suscetiveis à ação tóxica das diversas substâncias existentes no cigarro, hipótese que poderia ser testada em futuros estudos experimentais.

Em conclusão, apesar de tendência à aceleração da maturação placentária nas fumantes quando comparadas às não fumantes, não se evidenciou associação do tabagismo com aceleração da maturação placentária. As tabagistas tiveram aumento significante da impedância vascular nas artérias umbilicais na $32^{\mathrm{a}}$ semana, sem alteração na impedância das artérias uterinas.

4. Naeye RL, Peters EC. Mental development of children whose mothers smoked during pregnancy. Obstet Gynecol. 1984;64(5):601-7.

5. Müller JS, Antunes M, Behle I, Teixeira L, Zielinsky P. Acute effects of maternal smoking on fetal-placentalmaternal system hemodynamics. Arq Bras Cardiol. 2002; 78(2):148-55.

6. Pringle PJ, Geary MP, Rodeck CH, Kingdom JCP, Kayamba-Kay's S, Hindmarsh PC. The Hindmarsh PC. The influence of cigarette smoking on antenatal 
growth, birth size, and the insulin-like growth factor axis. J Clin Endocrinol Metab. 2005;90(5):2556-62.

7. Albuquerque CA, Smith KR, Johnson C, Chao R, Harding R. Influence of maternal tobacco smoking during pregnancy on uterine, umbilical and fetal cerebral artery blood flows. Early Hum Dev. 2004;80(1):31-42.

8. Grannum PA, Berkowitz RL, Hobbins JC. The ultrasonic changes in the maturing placenta and their relation to fetal pulmonic maturity. Am J Obstet Gynecol. 1979;133(8):915-22.

9. Brown HL, Miller JM Jr, Khawli O, Gabert HA. Premature placental calcification in maternal cigarette smokers. Obstet Gynecol. 1988;71(6 Pt 1):914-7.

10.Viggiano MGC, Barbacena ML, Jorge SD, Amaral WN. Tabagismo e maturação placentária precoce. J Bras Ginecol. 1991;101(9):365-8.

11.McKenna D, Tharmaratnam S, Mahsud S, Dornan J. Ultrasonic evidence of placental calcification at 36 weeks' gestation: maternal and fetal outcomes. Acta Obstet Gynecol Scand. 2005;84(1):7-10.

12.Vosmar MB, Jongsma HW, van Dongen PW. The value of ultrasonic placental grading: no correlation with intrauterine growth retardation or with maternal smoking. J Perinat Med. 1989;17(2):137-43.

13.Ates U, Ata B, Armagan F, Has R, Sidal B. Acute effects of maternal smoking on fetal hemodynamics. Int J Gynaecol Obstet. 2004;87(1):14-8.
14.Manning F, Walker D, Feyerabend C. The effect of nicotine on fetal breathing movements in conscious pregnant ewes. Obstet Gynecol. 1978;52(5):563-8.

15.Suzuki K, Minei LJ, Johnson EE. Effect of nicotine upon uterine blood flow in the pregnant rhesus monkey. Am J Obstet Gynecol. 1980;136(8):1009-13.

16.Castro LC, Allen R, Ogunyemi D, Roll K, Platt LD. Cigarette smoking during pregnancy: acute effects on uterine flow velocity waveforms. Obstet Gynecol. 1993;81(4):551-5.

17.Philipp K, Pateisky N, Endler M. Effects of smoking on uteroplacental blood flow. Gynecol Obstet Invest. 1984;17(4):179-82.

18.Andersen KV, Hermann N. Placenta flow reduction in pregnant smokers. Acta Obstet Gynecol Scand. 1984;63(8):707-9.

19.Asmussen I, Kjeldsen K. Intimal ultrastructure of human umbilical arteries. Observations on arteries from newborn children of smoking and nonsmoking mothers. Circ Res. 1975;36(5):579-89.

20. Pinto GR, Botelho C. Influência do tabagismo no sistema vascular materno-fetal: estudo em dopplervelocimetria. Rev Bras Ginecol Obstet. 2000;22(10):641-6.

21.Newnham JP, Patterson L, James I, Reid SE. Effects of maternal cigarette smoking on ultrasonic measurements of fetal growth and on Doppler flow velocity waveforms. Early Hum Dev. 1990;24(1):23-36. 